\title{
Nanocapsules: a novel formulation technology for platinum-based anticancer drugs
}

\author{
Irene HL Hamelers \& \\ Anton IPM de Kroon ${ }^{\dagger}$ \\ ${ }^{\dagger}$ Author for correspondence \\ Utrecht University, \\ Department Biochemistry of \\ Membranes, Bijvoet Institute \\ and Institute of \\ Biomembranes, Padualaan 8 , \\ 3584 CH Utrecht, \\ The Netherlands \\ Tel.: + 31302533424 ; \\ Fax: +31 302533 969; \\ a.i.p.m.dekroon@chem.uu.nl
}

\begin{abstract}
Platinum-based anticancer agents have been in widespread use for many years to successfully treat many different types of cancer. However, the efficacy of these drugs is limited by serious side effects. One of the strategies to reduce the side effects is encapsulation of the drug in a lipid formulation. Recently, a novel method for the efficient encapsulation of cisplatin in a lipid formulation was discovered. The method is unique in that it does not generate conventional liposomes but nanocapsules: small aggregates of solid cisplatin covered by a lipid bilayer. Carboplatin, a cisplatin-derived anticancer drug with different chemical properties, was also efficiently encapsulated by a similar method. The encapsulation in nanocapsules dramatically improves the in vitro cytotoxicity of the platinum drugs. The nanocapsule technology may be generally applicable in encapsulating platinum drugs with limited water solubility and low lipophilicity, and thereby improve the therapeutic index and profile of these drugs.
\end{abstract}

\section{Cisplatin in cancer treatment}

Rosenberg's discovery in 1965 that cis-diamminedichloroplatinum[II] (cisplatin, Figure 1A) caused filamentous growth of Escherichia coli $[1]$ was the prelude to testing cisplatin as an anticancer agent. Ever since, it has been in widespread use to successfully treat several forms of cancer, including testicular, ovarian, cervical, head and neck, and non-small-cell lung cancer [2].

Cisplatin is administered intravenously and once inside a cell, is hydrolyzed to form the aquated species, $[\mathrm{Pt}(\mathrm{NH} 3) 2 \mathrm{Cl}(\mathrm{OH} 2)]^{+}$and $[\mathrm{Pt}(\mathrm{NH} 3) 2(\mathrm{OH} 2) 2]^{2+}$. The low intracellular concentration of chloride ions facilitates this process. Compared with the dichloro species, the aquated species are more reactive to cellular targets that contain nucleophilic sites such as DNA, RNA, proteins, membrane phospholipids and thiol-containing molecules [3-7]. The primary biological target of the drug is DNA: approximately $1-5 \%$ of the total platinum taken up by the cells binds to DNA [8]. The platinum atom of cisplatin forms adducts with the $\mathrm{N}^{7}$ positions of purine bases, giving rise to primarily 1,2- or 1,3-intrastrand crosslinks, and to a relatively lower number of interstrand crosslinks [9]. The stable Pt-DNA adducts interfere with transcription and replication, and trigger apoptosis, leading to the death of the (cancer) cell [10].

The treatment of cancer with cisplatin is limited by side effects, including nausea, vomiting, nephrotoxicity, ototoxicity and peripheral neuropathy [11]. Nephrotoxicity can be managed by pre- and post-treatment hydration combined with the use of diuretics, as well as by serotoninreceptor antagonists [12]. Neurotoxicity, however, is still a significant dose-limiting side effect of cisplatin, which is manifested by peripheral neuropathy, tinnitus and high-frequency hearing loss [11]. The clinical use of cisplatin is limited further by extensive binding to plasma and tissue proteins, which leads to a rapid loss of bioavailability, and to inactivation of a large part of the administered dose (up to $96 \%$ within $24 \mathrm{~h}$ ) [13,14]. In addition to the side effects and the rapid inactivation of cisplatin, inherent and acquired resistance limits application [15]. The mechanisms that account for this drug resistance have been the subject of intensive study. It now appears that multiple pathways contribute, and that the primary mechanism may vary between the different model systems investigated so far, that is, (human) carcinoma cell lines and xenografts in nude mice (reviewed in [16]). Mechanisms thus far described include decreased accumulation of platinum in the cells through decreased uptake or increased efflux, increased DNA-repair, increased tolerance for the platinum-DNA adducts, and failure of apoptotic pathways (reviewed in [2]).

\section{Cisplatin analogues}

The severe side effects of cisplatin prompted a search for less toxic platinum analogues [17]. It was hypothesized that the modification of cisplatin to contain less labile leaving groups would alter its cytotoxicity. This led to the development 
Figure 1. Cisplatin (A), carboplatin (B), and oxaliplatin (C).

A<smiles>N[PH](N)(Cl)Cl</smiles>

B

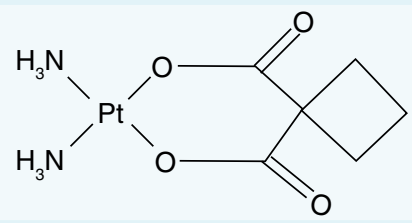

C

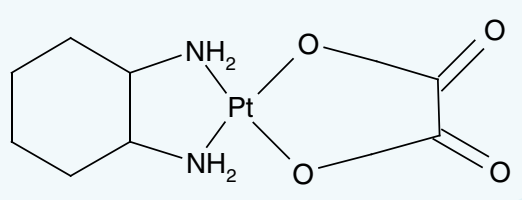

of cis-diammine-cyclobutane-1,1-dicarboxylatoplatinum[II] (carboplatin, Figure 1B) [17]. The leaving group of carboplatin confers good aqueous solubility and greater stability because it forms a six-membered ring with the platinum atom [18]. The substitution of the chloride leaving groups for cyclobutanedicarboxylate changed the reactivity considerably and thereby reduced serum protein binding. It was shown that this resulted in diminished side-effects on the kidney and the nervous system, and caused less nausea and vomiting, while antitumor activity was retained [19]. However, adverse hematological effects, in particular the occurrence of myelosuppression, are more frequent with carboplatin than with cisplatin, and dose limiting in the treatment of patients [20]. Moreover, the problems of intrinsic and acquired cisplatin resistance persist [15].

Since the discovery of cis- and carboplatin, thousands of additional platinum analogues have been synthesized and screened for anticancer activity in an attempt to overcome the limitations, and to broaden the range of treatable tumors. Among these compounds, only a few have been approved for use in the clinic, including 1,2-diamino-cyclohexane-(oxalato)platinum[II] (oxaliplatin) (Figure 1C), for the treatment of colorectal cancer [21,22]. An advantage of using oxaliplatin is that it can overcome cisplatin resistance in several but not all tested tumors [23]. Although cisplatin and oxaliplatin form the same types of adduct at the same sites

on the DNA, the adduct structures are distinct and are differentially recognized by some damage-recognition and mismatch-repair proteins. These disparate recognition and processing events are thought to contribute to differences in cytotoxicity and differences in the range of anticancer activities exhibited by oxaliplatin and cisplatin [24]. Oxaliplatin treatment, however, also results in detrimental side effects: hematologic toxicity, gastrointestinal tract toxicity and neuropathy [25].

In recent years, three new platinum compounds have been introduced in the clinic in Asia: nedaplatin (cis-diammineglycolatoplatinum[II]; [26]) lobaplatin (D-19466; 1,2-diammino-methylcyclobutaneplatinum[II]-lactate; [27]), and heptaplatin (SKI-2053R; cis-malonato-[4R,5R]-4,5,bis[aminemethyl]-2-isopropyl-1,3,-dioxolanplatinum[II]; [28]). Although they have some advantages over cisplatin (e.g., improved antitumor activity, a slightly different therapeutic range or an incomplete cross-resistance), they also induce serious dose-limiting toxicities and resistance in treated patients.

\section{Liposomal formulations of platinum drugs}

Another strategy to reduce systemic toxicity and prevent inactivation of platinum drugs in the bloodstream and tissue fluid is the encapsulation of the drug in a lipid formulation such as liposomes. Moreover, a lipid formulation may overcome platinum resistance by delivering a higher dose of the administered drug at the site of the tumor. Liposomes are micro-particulate or colloidal carriers, typically $0.05-0.5 \mu \mathrm{m}$ in diameter, which form spontaneously when certain lipids are hydrated. They are made of nontoxic and biodegradable material, and consist of an aqueous volume entrapped by one or more bilayers of natural and/or synthetic lipids. Systemic treatment with liposomes leads to extravasation and accumulation of liposomal drugs specifically within neoplastic tissues, because of the leaky vasculature and scarce lymphatic vessels in tumors [29]. Moreover, the liposome drug delivery platform offers the possibility of grafting tumor-specific ligands on the liposome membrane for active targeting to tumor cells, and potentially intracellular drug delivery [29].

Liposomally encapsulated cytotoxic drugs are becoming an established tool in the treatment of cancer. The liposomal anthracycline agents (doxorubicin and daunorubicin) have shown that these formulations can alter the efficacy and toxicity 
profiles of the parent compounds [30-32]. Following these initial successes, liposomal formulations of other agents (e.g., vincristine) have entered (pre)clinical trials [33,34], and liposomally encapsulated camptothecins [35,36] and topoisomerase inhibitors [37] are under investigation.

Several liposomal formulations of cisplatin have been developed. In SPI-077, cisplatin is encapsulated in a soluble form in sterically stabilized polyethylene glycol-coated (PEGylated or 'stealth'), $100 \mathrm{~nm}$ liposomes [38,39]. PEG is a physiologically stable water-soluble polymer that prevents the access of plasma proteins to the membrane surface by steric hindrance. Thus, it prevents aggregation and fusion of the liposomes and prolongs blood circulation [40,41]. Preclinical studies showed that SPI-077 exhibited an extended circulation time, increased antitumor efficacy and reduced toxicity compared with the free drug [38]. However, in Phase I and II studies, SPI-077 exhibited essentially no antitumor activity in patients [42-44]. A subsequent study in tumor-bearing mice indicated that although more SPI-077 distributes into tumors, it releases less Pt into the tumor, and forms fewer Pt-DNA adducts, as compared with free cisplatin [45].

Lipoplatin, another liposomal formulation of cisplatin [46] did exhibit antitumor efficacy in Phase I and II studies with reduced side effects compared with the free drug [47], and is currently being tested in Phase III studies. It may become the first lipid formulation of cisplatin to enter the clinic. Liposomal encapsulation of oxaliplatin by similar technology yielded Lipoxal, which was found to greatly reduce the side effects of oxaliplatin in a Phase I trial without losing efficacy [48].

Recently, multivesicular liposomes (MVLs) with an average diameter of $17 \mu \mathrm{m}$ containing cisplatin were tested in preclinical studies [49]. The therapeutic efficacy of the cisplatin-MVL preparation was reported to be significantly higher than that of cisplatin solution in S180 tumor-bearing mice [49]. Whether this formulation of cisplatin has antitumor activity in humans awaits future clinical studies.

\section{Cisplatin nanocapsules}

A major drawback of the aforementioned conventional liposomal formulations of cisplatin is the limited bioavailability of the drug in the tumor $[43,50]$. Key factors are likely the low water solubility $\left(8 \mathrm{mM}\right.$ at $\left.37^{\circ} \mathrm{C}\right)$ and low lipophilicity of cisplatin [51], which lead to liposomal formulations with relatively low drug-to-lipid molar ratios (in the order of 0.05-0.15). Burger et al. recently discovered an alternative method to encapsulate cisplatin in a lipid formulation with superior efficiency [52]. The method, which takes advantage of the limited solubility of the drug in water, produces cisplatin nanocapsules, nanoprecipitates of cisplatin that are surrounded by a lipid layer. These unique nanostructures are obtained by repeatedly freezing and thawing an equimolar dispersion of zwitterionic dioleoyl-phosphatidylcholine (DOPC) and anionic dioleoyl-phosphatidylserine (DOPS) in a concentrated, equilibrated aqueous solution of cisplatin. Nanocapsules have a characteristic bean shape with a typical width of $50 \mathrm{~nm}$ and a length between 50 and $250 \mathrm{~nm}$ (Figure 2). Importantly, the cisplatin nanocapsules have an unprecedented cisplatin-to-phospholipid molar ratio of 11:1, and an in vitro cytotoxicity up to 100-fold higher than the free drug (Figure 2) [52].

\section{Mechanism of formation \& molecular architecture of cisplatin nanocapsules}

The formation of cisplatin nanocapsules critically depends on the freeze-thaw cycles, and on the presence of negatively charged lipids. Instead of DOPS, the negatively charged phospholipids dioleoyl-phosphatidylglycerol (DOPG) and dioleoyl-phosphatidic acid (DOPA) can be used to prepare cisplatin nanocapsules [52,53]. The formation of nanocapsules is incompatible with high chloride concentration and alkaline $\mathrm{pH}$ [52], conditions that prevent the formation of the aqua species of cisplatin. These observations led to the proposal that electrostatic attraction between the positively charged aqua species and the negatively charged phospholipids is crucial for nanocapsule formation [52].

Using ${ }^{15} \mathrm{~N}$-labeled cisplatin, Chupin et al. [54] elucidated the molecular architecture of cisplatin nanocapsules by nuclear magnetic resonance (NMR) methods. Cisplatin nanocapsules were found to consist of a core of solid cisplatin virtually devoid of free water, coated by a phospholipid bilayer [54]. Ramachandran et al. [55] used atomic force microscopy (AFM) to characterize cisplatin nanoliposomes that were prepared according to a procedure that is very similar to the protocol for preparing nanocapsules. AFM force curves revealed that the nanoliposomes containing cisplatin are stiffer than vesicles not containing cisplatin, which was attributed to the presence of cisplatin clusters inside the liposomes [55], in agreement with previous data $[52,54]$. 


\section{Figure 2. Cytotoxicity of cisplatin nanocapsules and free} cisplatin towards IGROV1 human ovarian carcinoma cells.

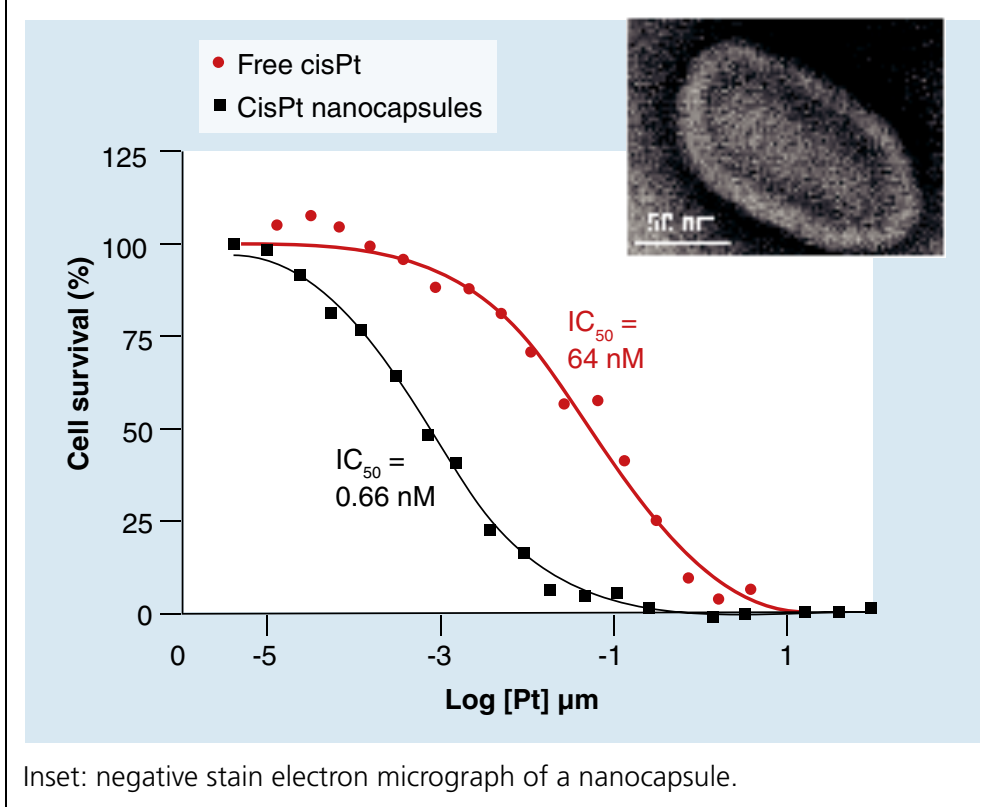

Magic angle spinning NMR complemented by mass spectrometry revealed the chemical composition of the nanocapsules' solid core, which was found to consist mainly of the dichloro species of cisplatin with a minor contribution of a newly identified positively charged chloride-bridged dimer [54]. Most likely the chloride-bridged dimer is formed as a result of the increasing concentrations of the dichloro and mono-aqua species in the remaining fluid during the freezing of water, which facilitates the reaction between these species [54]. The chloride-bridged dimer is not stable in the presence of water. Upon disrupting the lipid coat, cisplatin in the core returns to the initial equilibrium of dichloro and aqua species [54].

In addition to the bean shape and the solid core, the physical properties of the surrounding lipid bilayer distinguish nanocapsules from liposomes with a corresponding lipid composition. ${ }^{31}$ P-NMR revealed that the phospholipid headgroups in the bilayer coat of cisplatin nanocapsules are motionally restricted compared with liposomal lipids, with part of the lipids, most likely the DOPS molecules present in the inner leaflet, being fully immobilized [54]. These features were attributed to the strong electrostatic interaction between the negatively charged phosphatidylserine (PS) headgroup and the positively charged solid core. In addition, the interaction between the solid core and the stable coordination complex that forms between PS and cisplatin [5] could contribute to the restriction of motion [54].
The virtual absence of free water molecules in the nanocapsules implies that the bilayer coat tightly covers the solid cisplatin core. This places constraints on the flexibility of the bilayer coat that became apparent when the unsaturated phospholipids DOPC and DOPS were replaced by their saturated counterparts, which are commonly used in liposomal drug formulations. Whereas the unsaturated phospholipids are in the liquid crystalline phase at the temperature of nanocapsule formation, the saturated phospholipids are in the gel-state, and as a consequence they lack the flexibility to cover the cisplatin nanoprecipitates during the freeze-thaw cycles [53].

Based on the data summarized above, a model for the formation of nanocapsules as depicted in Figure 3 was proposed [52,54]. Cisplatin is concentrated in the residual fluid during freezing, giving rise to nanoprecipitates of dichloro-cisplatin that are covered by positively charged chloride-bridged dimers of cisplatin. Subsequently, the negatively charged DOPC/DOPS vesicles interact with the positively charged nanoprecipitates and reorganize to wrap them. The resulting nanocapsules do not dissolve upon thawing.

\section{Stability of cisplatin nanocapsules}

The stability of the nanocapsules is crucial for a possible future clinical application, and should therefore be optimized before animal testing. The DOPC/DOPS cisplatin nanocapsules suspended in water release their contents in a temperature-dependent way [53]. While stable at $4^{\circ} \mathrm{C}$, they lose their contents with a half time of $6.5 \mathrm{~h}$ at $37^{\circ} \mathrm{C}$. Interestingly, the formulations containing other anionic lipids were less stable. For example, the DOPC/DOPG formulation in water rapidly released all of its contents within $2 \mathrm{~h}$ at $37^{\circ} \mathrm{C}$. As PS forms a stable coordination complex with cisplatin whereas PG does not [5], the cisplatin-PS complex was suggested to play a role in nanocapsule stability. By varying the nanocapsules' content of cisplatin-PS complex, it was shown that the presence of cisplatin-PS complex reduces the rate of release of contents [53]. Conceivably, the initially electrostatic interaction of the solid core of the nanocapsules with the bilayer is stabilized as the cisplatin-PS coordination complex forms. In addition, it is possible that complex formation influences the permeability properties of the bilayer coat by affecting the acyl chain order. 
Upon resuspension in mouse serum, the DOPC/DOPS nanocapsules immediately collapsed, irrespective of the inclusion of $40 \%$ $(\mathrm{mol} / \mathrm{mol})$ of cholesterol in the formulation to reduce membrane permeability [53]. The presence of the negatively charged PS, a prerequisite for the formation of nanocapsules, is most likely the cause for the instability in serum. Although part of the PS reacts with cisplatin to form the neutral cisplatin-PS coordination complex, the remaining negative surface charge [52] probably leads to high-affinity binding of serum proteins to the bilayer coat, destabilizing the nanocapsules.

Coating nanocapsules with PEG shields the surface charge, thus reducing the binding of proteins [41]. Upon incorporation of 1,2-distearoyl-sn-glycero-3-phosphoethanolamine- $N$ (poly[ethyleneglycol]2000) [DSPE-PEG2000] up to $6 \mathrm{~mol} \%$ of total phospholipid content, cisplatin nanocapsules were obtained with a yield, cisplatin-to-phospholipid molar ratio, and morphology indistinguishable from DOPC/DOPS nanocapsules [53]. The stability of the cisplatin nanocapsules in serum was drastically improved by incorporating $6 \mathrm{~mol} \%$ of DSPE-PEG2000. The in vitro cytotoxicity of the PEGylated nanocapsules was comparable to that of the standard DOPC/DOPS nanocapsules. It is expected that the increase in stability will be beneficial in in vivo experiments. A further increase of the DSPE-PEG content destabilized the nanocapsules, possibly by impairing the interaction between the cisplatin core and the bilayer coat [53], due to steric hindrance and/or the presence of polyethylene glycol hydration water [39]. Cisplatin nanocapsules containing 6 mol\% DSPE-PEG will serve as the starting formulation for in vivo studies addressing the antitumor efficacy of cisplatin nanocapsules in tumor-bearing mice.

\section{Figure 3. Model for the mechanism of nanocapsule formation by freeze-thawing.}

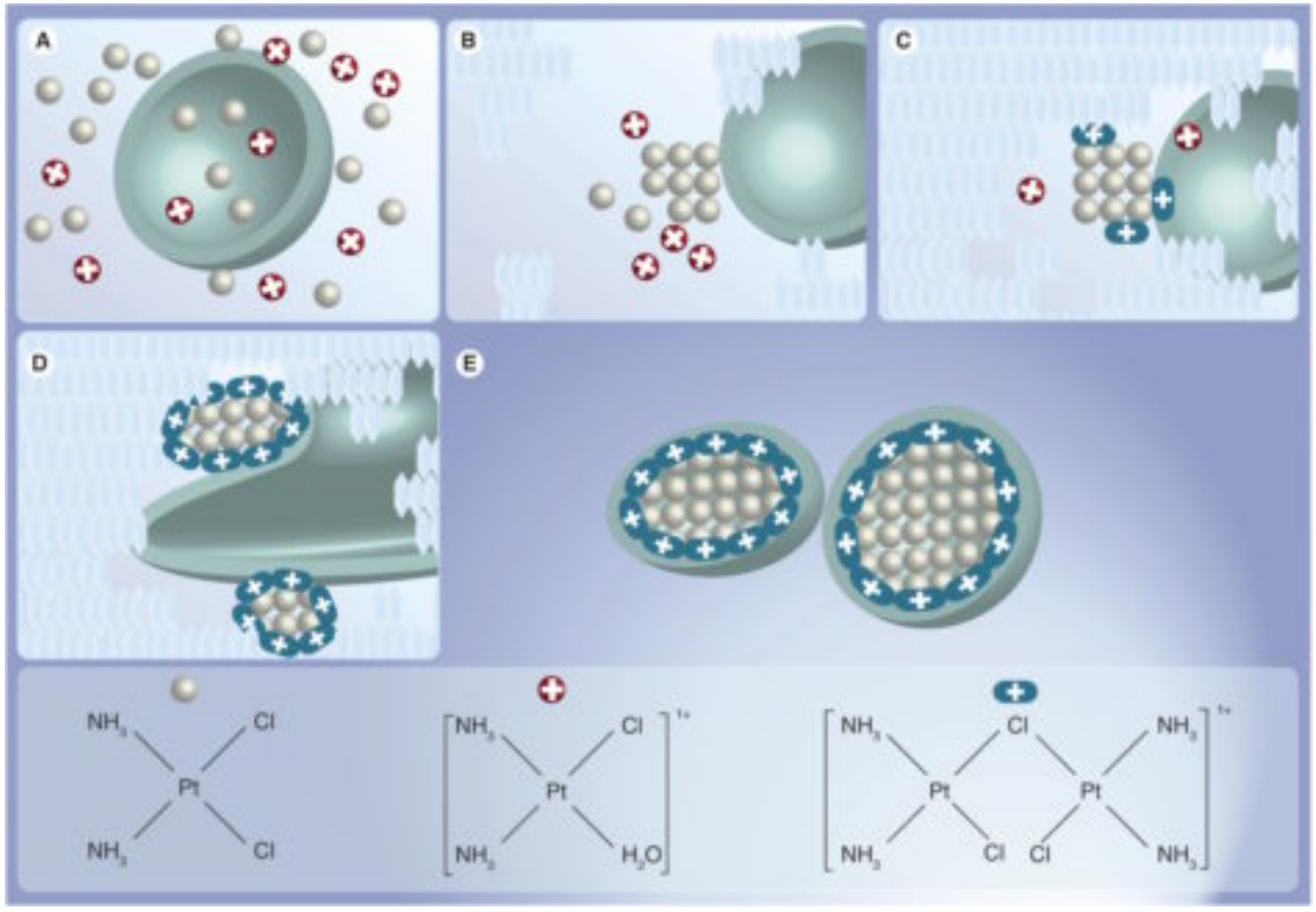

Partial hydrolysis of cisplatin in water yields the positively charged mono-aquated species of cisplatin. Neutral and positively charged species of cisplatin are present in a suspension of negatively charged liposomes before freeze-thawing (A). The formation of ice results in aggregation of the neutral cisplatin species $\mathbf{( B )}$, in the formation of the positively charged chloride-bridged dimers and their co-aggregation with the cisplatin nanoprecipitates (C), followed by interaction of the positively charged cisplatin nanoprecipitates with the negatively charged liposomes (D), that after thawing yields bilayer-encapsulated aggregates (nanocapsules) of cisplatin (E). 


\section{Carboplatin nanocapsules}

Encapsulation in nanocapsules promises to improve the therapeutic index and range of cisplatin. However, it was not immediately evident that the method for preparing nanocapsules could be applied to other platinum-based drugs with different properties, such as carboplatin. To test the applicability of the new nanocapsule technology to other platinum-based anticancer drugs, Hamelers et al. attempted to encapsulate carboplatin in nanocapsules [56]. Carboplatin is chemically distinct from cisplatin:

- The molecule is overall more hydrophobic owing to the substitution of the chloride leaving groups by cyclobutanedicarboxylate (Figure 1)

- Carboplatin is fivefold more water-soluble than cisplatin $[19,51]$

- The rate of hydrolysis of carboplatin is much slower [57]

When the initial protocol developed for cisplatin [58] was applied to carboplatin with some minor modifications, a lipid formulation enriched in carboplatin was obtained [56].

The encapsulation in nanocapsules strongly improves the cytotoxicity of carboplatin towards ovarian, renal and non-small-cell lung carcinoma cells in vitro [56]. The improved cytotoxicity of the carboplatin nanocapsules was found to be due to an increase in cellular accumulation of the drug after treatment with the nanocapsules as compared with the accumulation in cells treated with free carboplatin [56]. Like the formation of cisplatin nanocapsules, the formation of carboplatin nanocapsules strictly depends on the freeze-thaw steps and the presence of negatively charged lipids [56]. However, there are also some obvious differences in the nanocapsule formulations of cisplatin and carboplatin: the drug-to-lipid molar ratio of the carboplatin nanocapsules is much lower $(0.72: 1 ;[56])$ than that of the cisplatin nanocapsules $(11: 1 ;$ [52]). Recent data suggest that this difference in drugto-lipid molar ratio is also reflected in a difference in the structure of the particles (I.H.L. Hamelers, Unpublished Results). Moreover, the carboplatin nanocapsules are much more stable in water and in mouse serum compared with the cisplatin nanocapsules (I.H.L. Hamelers, Unpublished Results). In addition, the content of negatively charged lipids in the formulation of carboplatin could be reduced to at least $20 \%$ without a loss of yield or cytotoxicity [56], which is advantageous in view of clinical applications.

\section{Conclusion \& future perspective}

The formation and action of cisplatin and carboplatin nanocapsules show some remarkable parallels. Freeze-thawing and negatively charged lipids are required for formation, and the resulting lipid formulations contain an encapsulated drug concentration that exceeds the solubility limit of the drug [52,56]. These findings suggest that carboplatin and cisplatin nanocapsules are formed via similar mechanisms, despite the obvious differences in chemical structure, aquation rate and solubility in water between the two drugs. Future studies will address the molecular requirements for encapsulating (platinum) compounds in nanocapsules. In this context, the role of PS and the formation of platinum-PS coordination complexes deserve special attention. Both the cisplatin and the carboplatin nanocapsule formulations are extremely cytotoxic in vitro [52,56]. Moreover, cisplatin nanocapsules were reported to bypass the resistance of a cisplatin-resistant cell line with impaired cisplatin influx [59]. The mechanism(s) underlying the strongly enhanced cytotoxicity of the nanocapsules including the route of entry into cells is under investigation.

The most important challenge facing us is to translate the nanocapsules to formulations for clinical use. Current research is directed towards optimizing the nanocapsule preparations in terms of size, surface charge and stability, in order to address the antitumor efficacy in tumorbearing mice. With their unsurpassed encapsulation efficiency, nanocapsules present a promising new drug delivery system for (platinum) anticancer drugs with limited water solubility and low lipophilicity that may improve the therapeutic index and profile of these drugs.

\section{Acknowledgements}

We thank Drs. Koert Burger, Vladimir Chupin, Maria Velinova and Mr. Rutger Staffhorst for their contributions to the research on nanocapsules, and Prof. Dr. Ben de Kruijff for critical reading of the manuscript. This research was supported by the Dutch Cancer Society (KWF Kankerbestrijding) and by NanoNed, a national nanotechnology program coordinated by the Dutch Ministry of Economic Affairs. 
Executive summary

\section{Platinum drugs in cancer treatment}

- Cisplatin has been in widespread use to successfully treat several forms of cancer, including testicular, ovarian, cervical, head and neck, and non-small-cell lung cancer.

- Cisplatin forms stable Pt-DNA adducts that interfere with transcription and replication, and trigger apoptosis leading to the death of the (cancer) cell.

- Treatment of cancer with cisplatin is limited by side effects, rapid inactivation in the bloodstream and the occurrence of inherent and acquired resistance.

- Carboplatin and oxaliplatin are cisplatin analogues with reduced side effects and a different therapeutic range, respectively.

\section{Liposomal formulations of platinum drugs}

- To reduce systemic toxicity and to prevent inactivation of platinum drugs in the bloodstream and tissue fluid, the compounds have been encapsulated in liposomes.

- A major drawback of the conventional liposomal formulations of cisplatin is the limited bioavailability of the drug in the tumor. Key factors are likely to be the low water solubility and low lipophilicity of cisplatin, leading to liposomal formulations with low encapsulation efficiencies.

\section{Cisplatin nanocapsules}

- An alternative method to encapsulate cisplatin in a lipid formulation with superior efficiency is based on the repeated freezethawing of an equimolar dispersion of zwitterionic dioleoyl-phosphatidylcholine (DOPC) and anionic dioleoyl-phosphatidylserine (DOPS) in a concentrated, equilibrated aqueous solution of cisplatin.

- The method produces cisplatin nanocapsules, bean-shaped nanoprecipitates of cisplatin covered by a single lipid bilayer, with an unprecedented cisplatin-to-phospholipid molar ratio of 11:1, and exhibiting an in vitro cytotoxicity up to 100-fold higher than the free drug.

- Formation of cisplatin nanocapsules critically depends on the freeze-thaw cycles, and on the presence of negatively charged lipids. Electrostatic interaction between the positively charged cisplatin species and the negatively charged phospholipids is proposed to be crucial for nanocapsule formation.

- The stability of cisplatin nanocapsules in mouse serum has been drastically improved by incorporation of 1,2-distearoyl-sn-glycero3-phosphoethanolamine- $N$-[poly(ethyleneglycol)2000] (DSPE-PEG2000), setting the stage for addressing the antitumor efficacy of cisplatin nanocapsules in tumor-bearing mice.

\section{Carboplatin nanocapsules}

- Although carboplatin is chemically distinct from cisplatin, it can be encapsulated in nanocapsules that exhibit a strongly enhanced cytotoxicity towards ovarian, renal and non-small-cell lung carcinoma cells in vitro, compared with the free drug.

\section{Future perspective}

- With their high encapsulation efficiency, nanocapsules present a promising new drug delivery system for (platinum) anticancer drugs with limited water solubility and low lipophilicity that may improve the therapeutic index and profile of these drugs.

\section{Bibliography}

1. Rosenberg B, Vancamp L, Krigas T: Inhibition of cell division in Escherichia coli by electrolysis products from a platinum electrode. Nature 205, 698-699 (1965).

2. Wang D, Lippard SJ: Cellular processing of platinum anticancer drugs. Nat. Rev. Drug Discov. 4, 307-20 (2005).

3. Douple EB: cis-Diamminedichloroplatinum(II): effects of a representative metal coordination complex on mammalian cells. Pharmacol. Ther. 25, 297-326 (1984).

4. Speelmans G, Sips WH, Grisel RJ et al:: The interaction of the anticancer drug cisplatin with phospholipids is specific for negatively charged phospholipids and takes place at low chloride ion concentration. Biochim. Biophys. Acta 1283, 60-66 (1996).
5. Speelmans G, Staffhorst RW, Versluis K, Reedijk J, de Kruijff B: Cisplatin complexes with phosphatidylserine in membranes. Biochemistry 36, 10545-10550 (1997).

6. el-Khateeb M, Appleton TG, Gahan LR, Charles BG, Berners-Price SJ, Bolton AM: Reactions of cisplatin hydrolytes with methionine, cysteine, and plasma ultrafiltrate studied by a combination of HPLC and NMR techniques. J. Inorg. Biochem. 77, 13-21 (1999).

Reedijk J: New clues for platinum antitumor chemistry: kinetically controlled metal binding to DNA. Proc. Natl Acad. Sci. USA 100, 3611-3616 (2003).

8. Akaboshi M, Kawai K, Maki H, Akuta K, Ujeno Y, Miyahara T: The number of platinum atoms binding to DNA, RNA and protein molecules of HeLa cells treated with cisplatin at its mean lethal concentration. Jpn J. Cancer Res. 83, 522-526 (1992).
Fichtinger-Schepman AM, Lohman PH, Berends F, Reedijk J, van Oosterom AT: Interactions of the antitumour drug cisplatin with DNA in vitro and in vivo. IARC Sci. Publ. 83-99 (1986).

10. Dijt FJ,Fichtinger-Schepman AM, Berends F, Reedijk J: Formation and repair of cisplatin-induced adducts to DNA in cultured normal and repair-deficient human fibroblasts. Cancer Res. 48, 6058-6062 (1988).

11. Cooley ME, Davis LE, DeStefano M, AbrahmJ: Cisplatin: a clinical review. Part ICurrent uses of cisplatin and administration guidelines. Cancer Nurs. 17, 173-184 (1994).

Fillastre JP, Raguenez-Viotte G: Cisplatin nephrotoxicity. Toxicol. Lett. 46, 163-175 (1989). 
13. Ma J, Verweij J, Kolker HJ, van Ingen HE, Stoter G, Schellens JH: Pharmacokineticdynamic relationship of cisplatin in vitro: simulation of an intravenous. bolus and $3 \mathrm{~h}$ and $20 \mathrm{~h}$ infusion. Br. J. Cancer 69 , 858-862 (1994).

14. Schellens JH, Ma J, Planting AS et al: Relationship between the exposure to cisplatin DNA-adduct formation in leucocytes and tumour response in patients with solid tumours. Br. J. Cancer 73, 1569-1575 (1996).

15. Wernyj RP, Morin PJ: Molecular mechanisms of platinum resistance: still searching for the Achilles' heel. Drug Resist. Updat. 7, 227-232 (2004).

16. Siddik ZH: Cisplatin: mode of cytotoxic action and molecular basis of resistance. Oncogene 22, 7265-7279 (2003).

17. Connors TA, Jones M, Ross WC, Braddock PD, Khokhar AR, Tobe ML: New platinum complexes with anti-tumour activity. Chem. Biol. Interact. 5, 415-424 (1972).

18. Bloemink MJ, Reedijk J: Cisplatin and derived anticancer drugs: mechanism and current status of DNA binding. Met. Ions Biol. Syst. 32, 641-685 (1996).

19. Harrap KR: Preclinical studies identifying carboplatin as a viable cisplatin alternative. Cancer Treat. Rev. 12(Suppl. A), 21-33 (1985).

20. van der Vijgh WJ: Clinical pharmacokinetics of carboplatin. Clin. Pharmacokinet. 21, 242-261 (1991).

-21. Extra JM, Espie M, Calvo F, Ferme C, Mignot L, M Marty: Phase I study of oxaliplatin in patients with advanced cancer. Cancer Chemother. Pharmacol. 25, 299-303 (1990).

22. Extra JM, Marty M, Brienza S, Misset JL: Pharmacokinetics and safety profile of oxaliplatin. Semin. Oncol. 25, 13-22 (1998).

23. Barnes KR, Lippard SJ: Cisplatin and related anticancer drugs: recent advances and insights. Met. Ions Biol. Syst. 42, 143-177 (2004).

24. Mishima M, Samimi G, Kondo A, Lin X, Howell SB: The cellular pharmacology of oxaliplatin resistance. Eur. J. Cancer 38, 1405-1412 (2002).

25. Cassidy J, Misset JL: Oxaliplatin-related side effects: characteristics and management. Semin. Oncol. 29, 11-20 (2002).

26. Takigawa N, Segawa Y, Ueoka H et al.: Combination of nedaplatin and vindesine for treatment of relapsed or refractory nonsmall-cell lung cancer. Cancer Chemother. Pharmacol. 46, 272-278 (2000).
McKeage MJ, Higgins JD 3rd, Kelland LR: Platinum and other metal coordination compounds in cancer chemotherapy. A commentary on the sixth international symposium: San Diego, California, 23-26th January 1991. Br. J. Cancer 64(4), 788-792 (1991).

28. Kim NK, Kim TY, Shin SG et al: A Phase I study of $c i s$-malonato[(4R,5R)4,5-bis(aminomethyl)-1,3-dioxolane] platinum(II) in patients with advanced malignancies. Cancer 91, 1549-1556 (2001).

29. Gabizon AA, Shmeeda H, Zalipsky S: Pros and cons of the liposome platform in cancer drug targeting. J. Liposome Res. 16, 175-183 (2006).

30. Forssen EA, Male-Brune R, Adler-Moore JP et al: Fluorescence imaging studies for the disposition of daunorubicin liposomes (DaunoXome) within tumor tissue. Cancer Res. 56, 2066-2075 (1996).

31. Gabizon A, Tzemach D, Mak L, Bronstein M, Horowitz AT: Dose dependency of pharmacokinetics and therapeutic efficacy of pegylated liposomal doxorubicin (DOXIL) in murine models. J. Drug Target. 10, 539-548 (2002).

32. Gabizon AA: Pegylated liposomal doxorubicin: metamorphosis of an old drug into a new form of chemotherapy. Cancer Invest. 19, 424-436 (2001).

33. Hussein M: Pegylated liposomal doxorubicin, vincristine, and reduced-dose dexamethasone as first-line therapy for multiple myeloma. Clin. Lymphoma 4(Suppl. 1), S18-S22 (2003).

34. Leonetti C, Scarsella M, Semple SC et al: In vivo administration of liposomal vincristine sensitizes drug-resistant human solid tumors. Int. J. Cancer 110, 767-774 (2004).

35. Koshkina NV, Kleinerman ES, Waidrep C et al: 9-Nitrocamptothecin liposome aerosol treatment of melanoma and osteosarcoma lung metastases in mice. Clin. Cancer Res. 6, 2876-2880 (2000). et al: Feasibility, Phase I, and pharmacological study of aerosolized liposomal 9-nitro-20(S)camptothecin in patients with advanced malignancies in the lungs. Ann. NY Acad. Sci. 922, 352-354 (2000).

37. Colbern GT, Dykes DJ, Engbers C et al: Encapsulation of the topoisomerase I inhibitor GL147211C in pegylated (STEALTH) liposomes: pharmacokinetics and antitumor activity in HT29 colon tumor xenografts. Clin. Cancer Res. 4, 3077-3082 (1998).
8. Newman MS, Colbern GT, Working PK, Engbers C, Amantea MA: Comparative pharmacokinetics, tissue distribution, and therapeutic effectiveness of cisplatin encapsulated in long-circulating, pegylated liposomes (SPI-077) in tumor-bearing mice. Cancer Chemother. Pharmacol. 43, 1-7 (1999).

39. Peleg-Shulman T, Gibson D, Cohen R, Abra R, Barenholz Y: Characterization of sterically stabilized cisplatin liposomes by nuclear magnetic resonance. Biochim. Biophys. Acta 1510, 278-291 (2001).

40. Miller CR, Bondurant B, McLean SD, McGovern KA, O’Brien DF: Liposome-cell interactions in vitro: effect of liposome surface charge on the binding and endocytosis of conventional and sterically stabilized liposomes. Biochemistry 37, 12875-12883 (1998).

41. Torchilin VP: Recent advances with liposomes as pharmaceutical carriers. Nat. Rev. Drug Discov. 4, 145-160 (2005).

42. Harrington KJ, Lewanski CR, Northcote AD et al: : Phase I-II study of pegylated liposomal cisplatin (SPI-077) in patients with inoperable head and neck cancer. Ann. Oncol. 12, 493-496 (2001).

43. Meerum Terwogt JM, Groenewegen G, Pluim D et al: : Phase I and pharmacokinetic study of SPI-77, a liposomal encapsulated dosage form of cisplatin. Cancer Chemother. Pharmacol. 49, 201-210 (2002).

44. Vail DM, Kurzman ID, Glawe PC et al: STEALTH liposome-encapsulated cisplatin (SPI-77) versus carboplatin as adjuvant therapy for spontaneously arising osteosarcoma (OSA) in the dog: a randomized multicenter clinical trial. Cancer Chemother. Pharmacol. 50, 131-136 (2002).

45. Zamboni WC, Gervais AC, Egorin MJ et al:: Systemic and tumor disposition of platinum after administration of cisplatin or STEALTH liposomal-cisplatin formulations (SPI-077 and SPI-077 B103) in a preclinical tumor model of melanoma. Cancer Chemother. Pharmacol. 53, 329-336 (2004).

46. Boulikas T: Low toxicity and anticancer activity of a novel liposomal cisplatin (Lipoplatin) in mouse xenografts. Oncol. Rep. 12, 3-12 (2004).

47. Stathopoulos GP, Boulikas T, Vougiouka M et al:: Pharmacokinetics and adverse reactions of a new liposomal cisplatin (Lipoplatin): Phase I study. Oncol. Rep. 13, 589-595 (2005).

48. Stathopoulos GP, Boulikas T, Kourvetaris A, Stathopoulos J: Liposomal oxaliplatin in the treatment of advanced cancer: a Phase I study. Anticancer Res. 26, 1489-1493 (2006). 
49. Xiao C, Qi X, Maitani Y, Nagai T: Sustained release of cisplatin from multivesicular liposomes: potentiation of antitumor efficacy against $\mathrm{S} 180$ murine carcinoma. J. Pharm. Sci. 93, 1718-1724 (2004).

50. Bandak S, Goren D, Horowitz A, Tzemach D, Gabizon A: Pharmacological studies of cisplatin encapsulated in longcirculating liposomes in mouse tumor models. Anticancer Drugs 10, 911-920 (1999).

51. Riley CM, Sternson LA: Cisplatin. In: Analytical Profiles of Drug Substances. Florey K (Ed.). Academic Press, NY, USA 78-105 (1985).

-52. Burger KN, Staffhorst RW, de Vijlder HC et al:: Nanocapsules: lipid-coated aggregates of cisplatin with high cytotoxicity. Nat. Med. 8, 81-84 (2002)

53. Velinova MJ, Staffhorst RW, Mulder WJ et al:: Preparation and stability of lipidcoated nanocapsules of cisplatin: anionic phospholipid specificity. Biochim. Biophys. Acta 1663, 135-142 (2004).
54. Chupin V, de Kroon AI, de Kruijff B: Molecular architecture of nanocapsules, bilayer-enclosed solid particles of cisplatin. J. Am. Chem. Soc. 126, 13816-13821 (2004).

55. Ramachandran S, Quist AP, Kumar S, Lal R: Cisplatin nanoliposomes for cancer therapy: AFM and fluorescence imaging of cisplatin encapsulation, stability, cellular uptake, and toxicity. Langmuir 22, 8156-8162 (2006).

56. Hamelers IH, van Loenen E, Staffhorst RW, de Kruijff B, de Kroon AI: Carboplatin nanocapsules: a highly cytotoxic, phospholipid-based formulation of carboplatin. Mol. Cancer Ther. 5, 2007-2012 (2006).

57. Knox RJ, Friedlos F, Lydall DA, Roberts JJ: Mechanism of cytotoxicity of anticancer platinum drugs: evidence that cisdiamminedichloroplatinum(II) and cisdiammine- $(1,1-$ cyclobutanedicarboxylato)platinum(II) differ only in the kinetics of their interaction with DNA. Cancer Res. 46, 1972-1979 (1986).

58. de Kroon AI, Staffhorst RW, de Kruijff B, Burger KN: Cisplatin nanocapsules. Meth. Enzymol. 391, 118-125 (2005).
-59. Helleman J, Burger H, Hamelers IH et al.: Impaired cisplatin influx in an A2780 mutant cell line: Evidence for a putative, cis-configuration-specific, platinum influx transporter. Cancer Biol. Ther. 5, 943-949 (2006).

\section{Affiliations}

- Irene HL Hamelers

Utrecht University, Department Biochemistry of Membranes, Bijvoet Institute and Institute of Biomembranes, Padualaan 8, 3584 CH Utrecht, The Netherlands

Tel.: + 31302533345 ;

Fax: +31 302533969 ;

i.h.l.hamelers@chem.uu.nl

- Anton IPM de Kroon

Utrecht University, Department Biochemistry of Membranes, Bijvoet Institute and Institute of Biomembranes, Padualaan 8, 3584 CH Utrecht, The Netherlands Tel.: +31302533 424;

Fax: +31 302533 969;

a.i.p.m.dekroon@chem.uu.nl 